\title{
Hyperalgesia in spontaneous and experimental animal models of diabetic neuropathy
}

\author{
L. Wuarin-Bierman ${ }^{1}$, G. R. Zahnd ${ }^{1}$, F. Kaufmann ${ }^{2}$, L. Burcklen ${ }^{2}$ and J.Adler ${ }^{3}$ \\ ${ }^{1}$ Department of Medicine, University of Geneva and \\ ${ }^{2}$ Roche Research III, Basel, Switzerland; ${ }^{3}$ Hebrew University Hadassah Medical School, Jerusalem, Israel
}

\begin{abstract}
Summary. Hyperactivity of nociceptive C-fibers has been recently described in diabetic BB/Wistar rats. This study assesses the association of hyperalgesia, using an analgesy-meter, with elevated glycosylated haemoglobin levels in three animal models of diabetic and nutritional neuropathies: psammomys obesus (sand rat), streptozotocin-treated and galactose-fed rats. Pain threshold measurements (paw pressure test) and motor nerve conduction velocities were recorded in controls $(n=75)$, hyperinsulinaemic $(n=16)$, insulin-deficient ( $n=46)$ und galactosaemic ( $n=12)$ animals. The reproducibility of the paw pressure test, evaluated by a correlation coefficient, was statistically significant $(p<0.001)$. When compared with their controls $(396 \pm 18 \mathrm{~g})$, the average pain threshold in young diabetic sand rats $(309 \pm 17 \mathrm{~g})$ was found
\end{abstract}

to be markedly reduced and to correlate inversely $(p<0.001)$ with their respective HbA1c levels (mean 4.9 versus $7.4 \%$ ). Acute, subacute and chronic streptozotocin-diabetic rats displayed a reduction of pain threshold $(p<0.001)$ associated with slowed motor nerve conduction velocities $(p<0.001)$. Similarly, galactose-feeding over 4 weeks resulted in an elevation of glycosylated haemoglobin levels with significant $(p<0.001)$ reductions of pain threshold and motor nerve conduction velocity. It is concluded that hyperalgesia is a constant feature of sensory dysfunction in spontaneous and experimental models of diabetic neuropathy.

Key words: Pain, neuropathy, hyperglycaemia, sand rat, streptozotocin-diabetes, galactosaemia.
Neuropathy is a common complication of diabetes mellitus which subclinically may affect almost all patients $[1,2]$. Distal symmetrical polyneuropathy is the most frequent form and may be predominantly sensory, motor, autonomic or mixed [3]. Although the clinically worrisome lesions most often result in diminished perception of noxions stimuli, including pain, diabetic patients sometimes suffer from painful symptoms which may resolve after strict glycaemic control [4]. Experimental research on diabetic neuropathy is usually done on genetic or chemically induced diabetic animal models [5]. Diabetic neuropathy is generally evaluated by nerve conduction velocities [6-8] and certain biochemical abnormalities such as increased nerve polyol or decreased myo-inositol contents $[9,10]$.

This paper describes the use of the analgesy-meter (paw pressure test) as a tool for assessing sensory function in diabetic and galactosaemic rodents. Three animal models were studied. The gerbil psammomys obesus (sand rat) is an interesting model resembling non-insulin-dependent diabetes mellitus [11-13]. When in their natural habitat, the animals are lean and normoglycaemic. In captivity, however, given free access to regular laboratory chow they may become obese, hyperglycaemic, hyperinsulinaemic and often develop cataracts. Streptozotocin-diabetic rats are well known to develop an insulin-dependent diabetic syndrome [5, 7] with a concomitant decrease of motor and sensory nerve conduction velocities (NCV). Finally, galactosaemic rats represent a nutritional model of metabolic neuropathy. The accumulation of galactose by-products in peripheral nerves provides an insight into the role of the polyol pathway in the pathogenesis of diabetic neuropathy. This nutritional model is known to develop weight loss, elevated glycosylated haemoglobin levels and a decrease in NCV $[5,14,15]$.

Our results demonstrate a significant reduction in nociceptive pain thresholds in the three aforementioned models of neuropathy.

\section{Materials and methods}

\section{Animals}

Sand rats (psammomys obesus), were studied at the colony of Prof. Adler, Hebrew University-Hadassah Medical School, Jerusalem, Israel. Upon weaning, the animals were maintained on the two indicated standard diets. 


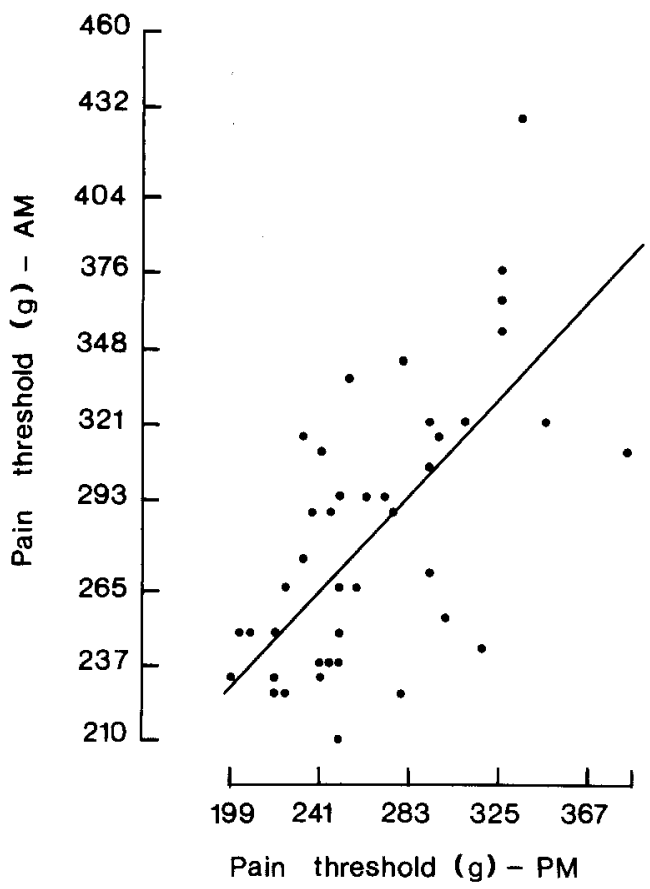

Figure 1. Correlation between morning and afternoon measurements of pain threshold in normal, streptozotocin-diabetic and galactosaemic rats $(r=0.64, p<0.0001)$

Male Wistar rats, aged 4-8 weeks, were given streptozotocin, galactose-fed or kept as controls. Among the streptozotocin-treated animals, one did not develop diabetes and another died.

Female Holtzmann rats, aged 6-8 weeks, received streptozotocin and were compared with age-matched controls.

Numbers of animals, details of treatment and time-course of the different studies are given below (experimental protocols).

\section{Analgesy-meter (paw pressure test)}

The analgesy-meter (Ugo Basile, Varese, Italy) is a simple instrument designed for rapid screening of drugs on the normal and inflamed rat paw. The test consists of applying a pressure to the hind paw, the force being increased at a constant rate. The paw is placed on a plith under a coneshaped pusher with a rounded tip. By pressing a pedal that activates a motor, the force increases on a linear scale. When the animal shows pain by withdrawing the paw, writhing and/or crying out, the pedal is released and the nociceptive pain threshold read on a scale. On rats, the force was applied to the first interdigital space; on psammomys obesus, because of morphological reasons, to the second metatarsian space. Each measurement is expressed in grams and represents the average value of the two hind paws. Pain tolerance thresholds were measured at least twice in all animals. Few individual measurements, differing by more than $2 \mathrm{SD}$ from the mean, were considered as unreliable when rats struggled (less than $10 \%$ of all the single measurements).

Reproducibility of the method was evaluated by a correlation coefficient of morning and afternoon threshold measurements in the streptozotocin-diabetic, galactosaemic and control groups after 4 weeks of treatment (Fig.1). The correlation between measurements is highly significant $(r=0.64 ; p<0.001)$. The reproducibility of the method was further assessed by comparing individual mean pain threshold values obtained in the morning and in the afternoon of the control group (t-test). The observed difference was not significant.

\section{Motor nerve conduction velocity (MNCV)}

Sciatic nerve conduction velocity was obtained from the left hind leg. The rats were anaesthetized with Nembutal (Abbott AG, Zug,
Switzerland, $50 \mathrm{mg} / \mathrm{kg}$ intraperitoneally). The stimulating electrodes were inserted at the sciatic notch and at the ankle. Muscle action potentials were recorded from the second interosseous muscle. Stimulation was supramaximal with a duration of $0.1 \mathrm{~ms}$. Stimulus and recording were provided by a Toennies digital myograph DA II (Toennies Erben KG, Freiburg, FRG). The distance between the stimulating points was measured and used to calculate motor nerve conduction velocity (MNCV) in $\mathrm{m} / \mathrm{s}$. Hindlimb temperature was kept on $36^{\circ} \mathrm{C}$ by means of a heating lamp. Limb temperature was recorded using a YSI telethermometer with a YSI thermistor probe inserted intramuscularly (Yellow Springs Instruments Co. Inc., Yellow Springs, Ohio, USA).

\section{Analytical methods}

Plasma glucose was measured enzymatically with a glucose II analyser (Beckman Instruments, Geneva, Switzerland). Plasma immunoreactive insulin levels were determined by radioimmunoassay (Amersham, International pic, Amersham, UK) using a human insulin standard. Glycosylated haemoglobin levels were performed by HPLC [17].

\section{Experimental protocols}

Psammomys obesus. We studied 50 animals which had been randomly fed one of two different diets after weaning. One control group ( $n=16,8$ males, 8 females) was fed a low calorie diet consisting of pellets containing $25 \%$ wheat straw reinforced with $3 \%$ casein complemented with 75\% Amrod chow [16]. The average age was $16.9 \pm$ 1 week. Among the pups which were kept on a standard (high-calorie) diet (Amrod 935 chow pellets) after weaning, 18 (11 males, 7 females) remained non-diabetic and 16 (10 males, 6 females) developed a diabetic syndrome within 2 months. The mean ages were $18 \pm 1.2$ weeks for the former and $13 \pm 0.6$ weeks for the latter.

Physical data include weight and paw pressure threshold measurements. Cataracts were assessed macroscopically. Full chemical data was obtained from tail blood on eight low-calorie-fed and all other sand rats. Data include fasted and non-fasted glycaemias, glycosylated haemoglobin values and immunoreactive insulin levels.

Streptozotocin-treated and galactose-fed rats. Group A consisted of male Wistar rats which were initially divided into three homogenous groups having identical mean weights and pain thresholds. Eighteen animals were injected subcutaneously with $70 \mathrm{mg} / \mathrm{kg}$ streptozotocin at 1 to 2 months of age. Fifteen animals were maintained on a standard $40 \%$ galactose diet and 15 served as a control group.

After 2 weeks of treatment, weights and nociceptive pressure thresholds were determined.

After 4 weeks of treatment the animals were evaluated for the following parameters: weight, glycaemia, glycosylated haemoglobin, immunoreactive insulin (IRI), MNCV and pain threshold.

Group B consisted of 17 female Holzmann rats who received streptozotocin as above with age- and sex-matched controls. Evaluation was made after 6 weeks of treatment.

Group C was composed of 11 male Wistar rats studied 31 weeks after $70 \mathrm{mg} / \mathrm{kg}$ streptozotocin injections and compared with agematched controls.

\section{Statistical analysis}

All numerical data are expressed as mean \pm SEM. Statistical comparisons were made using the unpaired Student's t-test. Relationships between the different variables were assessed by Pearson's simple correlation coefficient. 
Table 1. Physical and chemical data from psammomys obesus

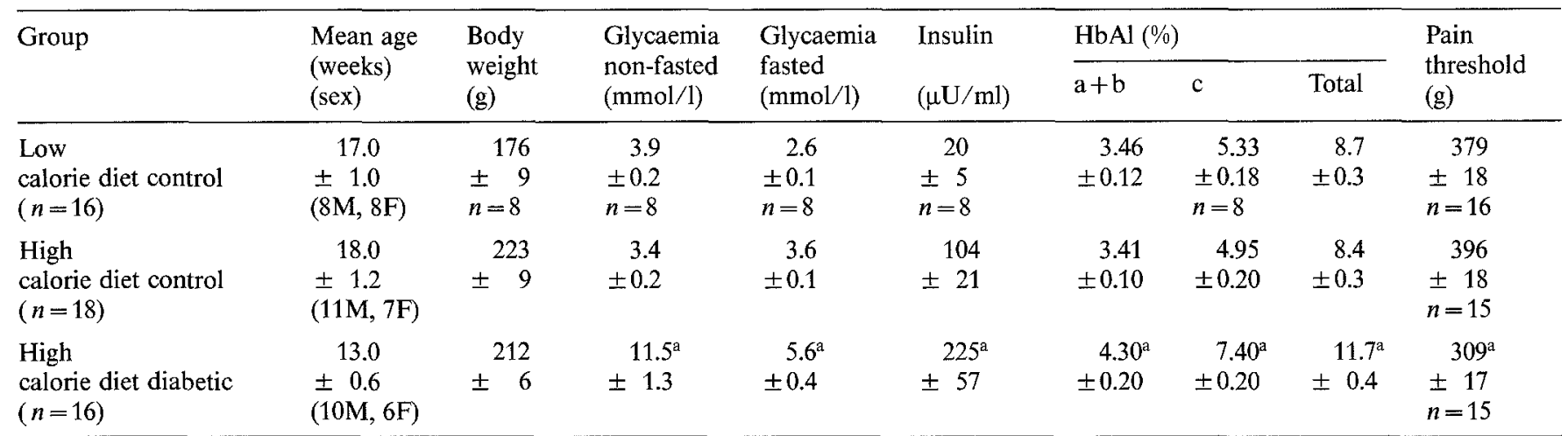

${ }^{a} p<0.01$ versus low and high caloric diet control groups. The animals, in families, were on an experimental feeding protocol when we had access to them. Although the groups seem unhomogeneous, no statistically significant difference existed in the data obtained from males and females. No correlation was found between nociceptive pressure thresholds and age or weight. Thus, the decrease in pain threshold is inherent to the diabetic state of the animals. Data are expressed as means $\pm \mathrm{SEM} ; \mathrm{M}=$ male; $\mathrm{F}=$ female

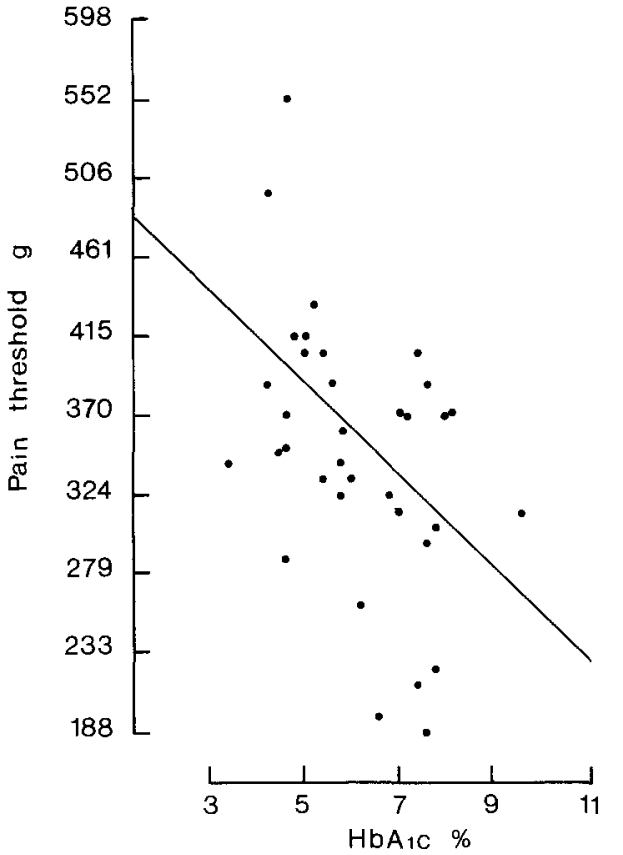

Figure 2. Relationship between pain threshold and HbAlc in normoglycaemic and diabetic sand rats $(r=0.43, p=0.005)$

\section{Results}

\section{Psammomys obesus}

The data obtained in the sand rats are shown in Table 1. The animals fed a low-calorie, non-diabetogenic diet, were lean, normoglycaemic and had low fasting plasma insulin levels. The average body weight was slightly lower (178 versus $223 \mathrm{~g}$ ) than that of the high-calorie controls. Glycosylated haemoglobin levels were similar in both control groups. Fasting IRI levels in the standard diet control group were significantly higher $(104 \pm 21 \mu \mathrm{U} / \mathrm{ml})$ than those in the low-calorie control group $(20 \pm 5 \mu \mathrm{U} / \mathrm{ml} ; p<0.01)$.
A diabetic syndrome appeared in a group of animals fed on a standard diet only. Their age was significantly $(p<0.05)$ younger than the control group and extrapolation of weight to age showed them to be overweight. Fasted and non-fasted glycaemias and glycosylated haemoglobin levels were significantly higher $(p<0.01)$ than either control group. Seven animals had developed macroscopically visible cataracts, but a slitlamp examination was not performed and detailed cataract assessment is thus unknown. The average pain threshold in the diabetic animals $(309 \pm 17 \mathrm{~g})$ was significantly lower $(p<0.01)$ than those of either the low or normal calorie groups $(379 \pm 18$ and $396 \pm 18$ respectively). Blood sugars were highly correlated with HbAlc levels $(r=0.7 ; p<0.001)$. A significant inverse correlation exists between pain threshold levels and HbAlc ( $r=0.43 ; p=0.001$; Fig. 2 ), but no correlation between pain thresholds and fasted glycaemias was found $(r=0.30 ; p=0.07)$. No correlation was found between weight and pain thresholds $(r=0.21 ; p=0.2)$ nor was there a dependency between threshold and age as assessed by linear regression analysis.

When diabetic and non-diabetic sand rats younger than 15 weeks of age were compared, a statistically significant difference in pain thresholds existed. These observations suggest that the decrease in pain thresholds cannot be attributed to the lower age nor the weight differences of the diabetic animals. Furthermore, no statistical difference existed between male and female values.

\section{Streptozotocin-diabetic rats}

After 2 weeks of treatment (group A), diabetic rats had a reduced body weight compared with control rats $(253 \pm 6 \mathrm{~g}$ versus $317 \pm 7 \mathrm{~g})$. Pain thresholds were reduced from $313 \pm 7 \mathrm{~g}$ to $243 \pm 6 \mathrm{~g}$. MNCV measurements were not performed.

At 4 weeks, weights decreased from $318 \pm 5 \mathrm{~g}$ in controls to $234 \pm 5 \mathrm{~g}$, non-fasted glycaemias increased 
Table 2. Physical and chemical data from rats, 4 weeks after streptozotocin-treatment and galactose-feeding

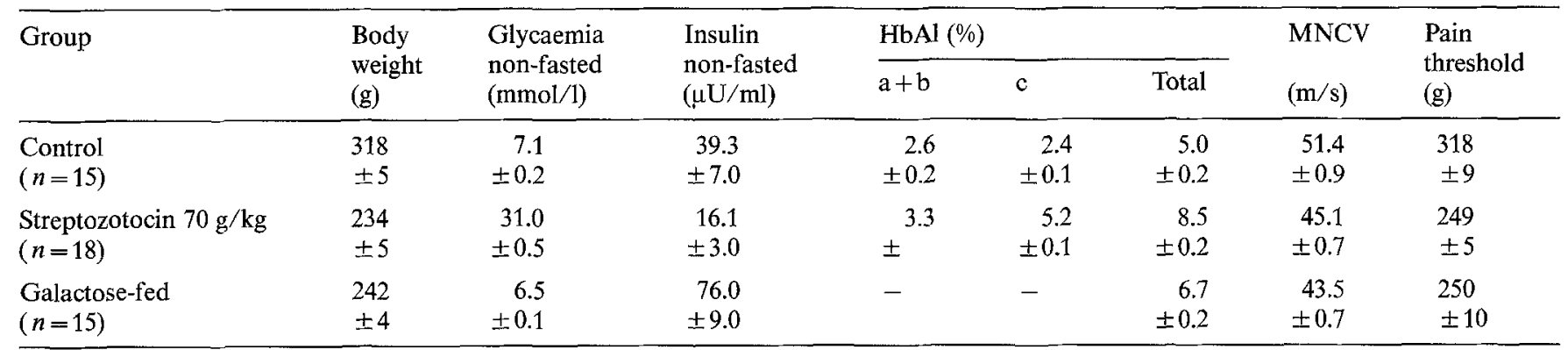

All values in streptozotocin-treated and galactose-fed groups are different $(p<0.001)$ from controls. Data are expressed as means \pm SEM

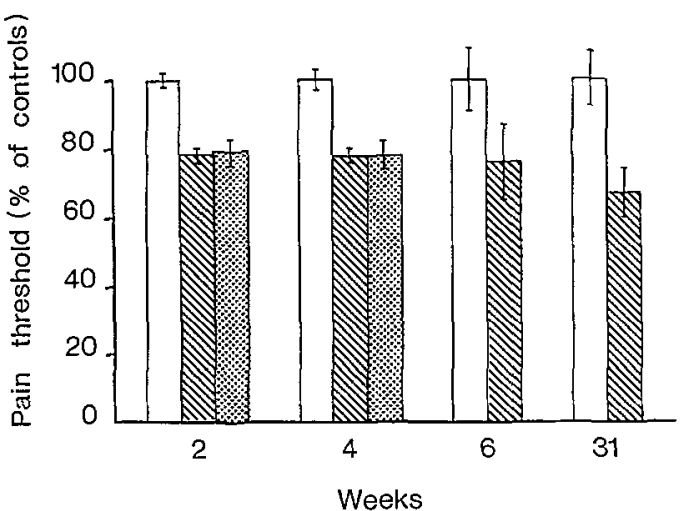

Figure 3. Effect of the duration streptozotocin-induced diabetes or galactosaemia 确 on hyperalgesia in the rat

from $7.1 \pm 0.2$ to $31 \pm 0.5 \mathrm{mmol} / 1$ and total $\mathrm{HbAl}$ rose to $8.5 \pm 0.2 \%$. MNCV decreased by $12.3 \%$ from $51.4 \pm$ $0.90 \mathrm{~m} / \mathrm{s}$ to $45.1 \pm 0.7 \mathrm{~m} / \mathrm{s}(p<0.001)$. Analgesymetry showed a reduction of pain thresholds from $318 \pm 9 \mathrm{~g}$ to $249 \pm 5 \mathrm{~g}$ (Table 2).

Group B at 6 weeks also had weight loss ( $265 \pm 3$ to $211 \pm 5 \mathrm{~g})$, hyperglycaemia $(27.7 \pm 0.5 \mathrm{mmol} / \mathrm{l})$ and decreased MNCV $(39.2 \pm 0.5$ versus $43.2 \pm 0.6 \mathrm{~m} / \mathrm{s})$. Pain thresholds were reduced from $297 \pm 9 \mathrm{~g}$ to $225 \pm 6 \mathrm{~g}$.

Group C, diabetic for 31 weeks when studied, had hyperglycaemia $(23.0 \pm 1.1 \mathrm{mmol} / \mathrm{l})$ and a markedly lower weight $(259 \pm 13 \mathrm{~g})$ than controls $(484 \pm 12 \mathrm{~g})$. The most important reduction of pain threshold was observed in this group: $221 \pm 5 \mathrm{~g}$ versus $334 \pm 13 \mathrm{~g}$ in controls. MNCV measurements were not performed.

All biochemical and physical parameters were significantly different from controls $(p<0.001)$. Pain thresholds showed a tendency to decrease as a function of the duration of the diabetic syndrome with reductions of $22 \%, 22 \%, 24 \%$ and $34 \%$ at $2,4,6$ and 31 weeks (Fig.3).

Correlation analysis performed on controls and streptozotocin-treated animals of group A showed a highly significant inverse relationship between pain thresholds and glycosylated haemoglobin levels $(r=0.76 ; p<0.001)$.

\section{Galactosaemic rats}

Weights of galactosaemic animals were $248 \pm 9 \mathrm{~g}$ after 2 weeks of treatment and $242 \pm 4 \mathrm{~g}$ after 4 weeks and were significantly $(p<0.001)$ lower than control values. Biochemical data at 4 weeks were as follows: glycaemia $6.5 \pm 0.1$ versus $7.1 \pm 0.2 \mathrm{mmol} / 1$ and total $\mathrm{HbAl}$ levels $6.7 \pm 0.2 \%$ versus $5 \pm 0.2 \%$. These results are significantly different $(p<0.001)$ from control and diabetic values (see above). Pain thresholds were reduced by 21 and $22 \%$ after 2 and 4 weeks of treatment (see Fig.3). After 4 weeks, MNCV were reduced to $43.5 \pm 0.7 \mathrm{~m} / \mathrm{s}$ versus $51.4 \pm 0.9 \mathrm{~m} / \mathrm{s}(p<0.001)$ (Table 2).

\section{Discussion}

In view of the diversity of diabetic syndromes associated with peripheral neuropathy, our study was performed on animal models presenting different abnormalities of pancreatic B-cell function or carbohydrate metabolism: a hyperinsulinaemic diabetic syndrome in the sand rat, an insulin-deficient diabetes in streptozotocin-treated rats and galactose-fed rats with normal insulin and glucose levels. As opposed to observations in sand rats, a potential toxic influence on neurological findings in streptozotocin-treated animals cannot be ruled out.

We have evaluated the use of paw pressure test for assessing sensory function in rodents. The method was shown to be reproducible (Fig. 1). Nociceptive pressure thresholds were significantly decreased in the three chosen animals models and were more pronounced with the duration of the diabetic syndrome. We found a highly significant inverse correlation between pain thresholds and glycosylated haemoglobin. Thus, the duration and severity of the diabetic syndrome are determinant in the genesis of this sensory dysfunction. We did not determine this correlation in galactose-intoxicated rats as they do not present true elevated glucosylated haemoglobin levels. However, increased non-enzymatic galactosylation of haemoglobin and other proteins have been shown to occur due to the physical properties of galactose [18]. 
MNCV were decreased by $10 \%$ to $12 \%$ in streptozotocin-diabetic rats and by $15 \%$ in galactosaemic rats. These results are very similar to those found by others, particularly Moore and colleagues [8], who extensively studied alloxan- and streptozotocin-diabetic rats and described reductions of 10 to $20 \%$ in several classes of sensory and motor nerve fibers. Preliminary electrophysiological studies have been performed on the isolated sciatic nerve from sand rats. The results showed a defect of nerve conduction velocities $(-26 \%)$ in the diabetic animals (personal communication, Dr. S.Constantini, Hadassah Hospital, Jerusalem). The majority of animal research on diabetic neuropathy is done on the chemical model or more recently on the spontaneously diabetic BB/Wistar rat. Much less is done on animal models of non-insulin-dependent diabetes such as the gentically mutant $\mathrm{db} / \mathrm{db}$ mouse $[5,19]$. Previous reports suggest that the sand rat may represent a useful model for the study of Type 2 (non-insulin-dependent) diabetes and its complications. The lower age of the diabetic sand rats in our study as compared to their control groups explains the absence of the expected weight gain in our results. However, we observed that they were phenotypically obese and had developed a characteristic "buffalo hump". No correlation between pain thresholds and age or weight were found and hyperalgesia can thus be attributed solely to the diabetic state of the animals.

Experimental galactosaemia was first developed as a model to study polyol accumulation in the genesis of diabetic complications. If this osmotic hypothesis seems to apply to diabetic cataract formation, there is actually little evidence for its role in the pathogenesis of polyneuropathy. However, non-enzymatic glycosylation of proteins in the pathogenesis of certain chronic complications of diabetes seems to be important [20], and thus galactosylation of nerve proteins could serve as a useful parameter for studying this aspect in the development of diabetic neuropathy.

The importance of chronic hyperglycaemia as a major factor in the etiology and electrophysiological features of diabetic neuropathy has been invoked in human [21-23] and in experimental diabetes [9, 24]. MNCV abnormalities in Type 1 (insulin-dependent) $[23,25,26]$ and Type 2 [22] diabetic patients have been related to metabolic control. Improvements in $\mathrm{MNCV}$ have been shown to occur with continuous subcutaneous insulin infusion (CSII). Sensory nerve conduction velocities, however, have been reported unaffected by short term CSII $[4,27]$ or moderately improved by 8 months of this treatment [28]. Nevertheless, near normal glucoregulation appears to provide subjective improvement, particularly pain relief, in patients with symptomatic polyneuropathy. It is of interest that acute painful neuropathy, related to hyperglycaemia and associated with severe weight loss, has been shown to resolve upon improved control of diabetes and weight gain with insulin [29].
The mechanisms responsible for the decreases in pain threshold levels are not yet elucidated. It may be located at the receptor level as animal studies have shown an altered response to opiate agonists and antagonists with altered pain thesholds measured with the tail-flick test in streptozotocin-diabetic rats [30, 31]. Morely and colleagues [32] found a decrease in pain threshold levels and maximal levels of pain tolerance in diabetic patients and suggested that glucose may modulate opioid receptors in man [32]. Another hypothesis could be damage to small unmyelinated nerve fibers (C-fibers) or A-nociceptive fibers. Indeed, Burchiel and colleagues [33] recently demonstrated that spontaneous hyperactivity of nociceptive afferent fibers is a frequent phenomenon in the diabetic BB/ Wistar rat. They conclude that hyperactivity in a nociceptive axon with a nonconducting distal segment would explain the coexistence of spontaneous pain in an anaesthetic region so often seen in neuropathic patients. Finally, damage to spinal inhibitory pathways or a combination of the above could explain the decreased pain threshold levels and neuropathic pain.

We have demonstrated two types of nerve dysfunction in our animal models: hyperalgesia and reduced MNCV. These two abnormalities do not seem to reflect the same impairment of neurofunction nor imply the same pathogenetic mechanism. Reservations about the relevance of motor conduction velocities to neuropathic symptoms have been expressed by others [34]. Indeed, the major neurological problems in diabetic patients are the sensory and autonomic and not the motor components of diabetic polyneuropathy.

In addition, our results indicate that the paw pressure test constitutes a simple and reproducible method for assessing sensory function in the rodent.

Acknowledgements. The authors are indebted to J.J.Goy, J.Derrien (Geneva), C. Lacoste (Basel), G. Lazarovici and M. Marton (Jerusalem) for excellent technical assistance and to Mrs. A.Volpé and I.Héritier for secretarial aid in preparation of the manuscript. This study was supported by Swiss National Science Foundation grant No. 3.840-0.83.

\section{References}

1. Noel P (1973) Sensory nerve conduction in the upper limits at various stages of diabetic neuropathy. J Neurol Neurosurg Psychiatry $36: 786-796$

2. Fraser DM, Campbell IW, Ewing DJ, Murray A, Neilson JMM, Clarke BF (1977) Peripheral and autonomic nerve function in newly diagnosed diabetes mellitus. Diabetes 26: 546-550

3. Brown MJ, Asbury AK (1984) Diabetic neuropathy. Ann Neurol 15: $2-12$

4. Boulton AJM, Drury J, Clarke B, Ward JD (1982) Continuous subcutaneous insulin infusion in the management of painful neuropathy. Diabetes Care 5: 386-390

5. Brown MR, Dyck PJ, McLearn GE, Sima AAF, Powell HC, Porte D Jr (1982) Central and peripheral nervous system complications. Diabetes 31 [Suppl 1]: 65-70

6. Gilliat RW, Willison RG (1962) Peripheral nerve conduction in diabetic neuropathy. J Neurol Neurosurg Psychiatry 25: 11-18 
7. Sharma AK, Thomas PK (1974) Peripheral nerve structure and function in experimental diabetes. J Neurol Sci 23: 1-15

8. Moore SA, Peterson RG, Felten DL, O'Connor BL (1980) A quantitative comparison of motor and sensory conduction velocities in short- and long-term streptozotocin- and alloxan-diabetic rats. J Neurol Sci 48: 133-152

9. Greene DA, Lattimer S, Ulbrecht J, Carrol P (1985) Glucose-induced alterations in nerve metabolism: current perspective on the pathogenesis of diabetic neuropathy and future directions for research and therapy. Diabetes Care 8: 290-299

10. Clements RS (1979) Diabetic neuropathy - new concepts in its etiology. Diabetes 28: 604-611

11. Adler JH, Hodis Y, Marton M, Lazarovic G (1978) Patterns of hyperglycaemia in sand rats. Biochem Med 20:315-322

12. Zahnd GR, Adler JH (1984) Sand rat as a model of diabetic cataract - a major blinding condition. In: Shafrir E, Renold AE (eds) Lessons from animal diabetes. John Libbey, London, pp 500-502

13. Marquié G, Duhault J, Jacotot B (1984) Diabetes mellitus in sand rats (psammomys obesus). Diabetes 33: 438-443

14. Ivor LP, Wolf PL, Castello ML, Powell HC (1979) Elevated haemoglobin Alc in experimental galactosaemia. Clin Chem 25: $1076 \mathrm{a}$

15. Engerman RL, Kern TS (1984) Experimental galactosaemia produces diabetic-like retinopathy. Diabetes 33:97-100

16. Adler JH, Lazarovici G, Marton M, Saleternik-Vardi R (1985) Nutrition of the sand rat (psammomys obesus) in relation to reproduction and diabetes. Isr J Med Sci 21: 769-771

17. Blanc MH, Rhie FH, Dunn PJ, Soeldner JS (1981) The determination of glycosylated haemoglobin in rats using high pressure liquid chromatography. Metabolism 30: 317-322

18. Bunn HF, Higgins PJ (1981) Reaction monosaccharides with proteins: possible evolutionary significance. Science 213: 222-224

19. Robertson DM, Sima AAF (1980) Diabetic neuropathy in the mutant mouse c57BL/ks (db/db). Diabetes 29:60-67

20. Kennedy L, Baynes JW (1984) Non-enzymatic glycosylation and the chronic complications of diabetes: an overview. Diabetologia 26: $93-98$

21. Pirart J (1977) Diabète et complications dégénératives. Présentation d'une étude prospective portant sur 4400 cas observés entre 1947 et 1973. Diab Metab 3: 97-107, 173-182, 245-256

22. Graf RJ, Malter JB, Halar E, Porte D Jr (1979) Nerve conduction abnormalities in untreated maturity-onset diabetes: relation to levels of fasting plasma glucose and glycosylated haemoglobin. Ann Intern Med 90: 298-303

23. Ward JD, Barnes CG, Fischer DJ, Jessop JD, Baker RWR (1971) Improvement in nerve conduction following treatment in newly diagnosed diabetics. Lancet 1: 428-431
24. Winegrad AI, Greene DA (1976) Diabetic polyneuropathy: the importance of insulin deficiency, hyperglycaemia and alterations in myo-inositol metabolism in its pathogenesis. N Engl $\mathrm{J}$ Med 295: 1416-1421

25. Young RJ, Ewing DJ, Clarke BF (1983) Nerve function and metabolic control in teenage diabetics. Diabetes 32:142-147

26. Boulton AJM, Knight G, Drury J, Ward JD (1985) The prevalence of symptomatic, diabetic neuropathy in an insulin-treated population. Diabetes Care 8: 125-128

27. Pietri A, Ehle AL, Raskin P (1980) Changes in nerve conduction velocity after 6 weeks of glucoregulation with portable insulin infusion pumps. Diabetes 29: 668-671

28. Service FJ, Rizza RA, Daube JR, O'Brien PG, Dyck PJ (1985) Near normoglycaemia improved nerve conduction and vibration sensation in diabetic neuropathy. Diabetologia 28: 722-727

29. Archer AG, Watkins PJ, Thomas PK, Sharma AK, Payan J (1983) The natural history of acute painful neuropathy in diabetes mellitus. J Neurol Neurosurg Psychiatry 46: 491-499

30. Simon GS, Dewey WL (1981) The effects of streptozotocin-induced diabetes on the antinociceptive potency of morphine. $\mathbf{J}$ Pharmacol Exp Ther 2: 318-323

31. Simon GS, Borzelleca J, Dewey WL (1981) Streptozotocin-induced diabetes selectively alters the potency of certain narcotic analgesics. Mechanism of diabetes: morphine interaction. $\mathbf{J}$ Pharmacol Exp Ther 218: 324-329

32. Morley GK, Mooradian AD, Levine AS, Morley JE (1984) Mechanism of pain in diabetic peripheral neuropathy. Am J Med 77: 79-82

33. Burchiel KJ, Russel LC, Lee RP, Sima AAF (1985) Spontaneous activity of primary afferent neurons in diabetic BB/Wistar rats. Diabetes 34: 1210-1213

34. Greene DA, Brown MJ, Braunstein SN, Schwartz SS, Asbury AK, Winegrad AI (1981) Comparison of clinical course and sequential electrophysiological tests in diabetics with symptomatic polyneuropathies and its implications for clinical trials. Diabetes 30: $139-147$

Received: 7 November 1986

and in revised form: 7 July 1987

Dr. Gaston Zahnd

Fondation pour recherches médicales

64 av. de la Roseraie

CH-1211 Geneva-4

Switzerland 